Published in final edited form as:

Nat Chem Biol. 2008 February ; 4(2): 86-88. doi:10.1038/nchembio0208-86.

\title{
Small molecule versus DNA repair nanomachine
}

\author{
James T Stivers \\ Department of Pharmacology and Molecular Sciences, Johns Hopkins Medical School, 725 North \\ Wolfe Street, Baltimore, Maryland 21205, USA. e-mail: jstivers@jhmi.edu
}

Abstract

The MRN protein megacomplex mediates repair of double-stranded DNA breaks (DSBs) by tethering together broken ends of chromosomes and signaling a cascade of events required for DNA repair. The first small-molecule inhibitor that disrupts MRN function provides a valuable new tool for functional studies of DSB repair in cells.

The efficient sensing of DSBs and the coordination of this detection event with cell cycle progression or apoptosis is the responsibility of the Mre11-Rad50-Nbs1 (MRN) protein complex. The central role of this complex in signaling the presence of DSBs and then coordinating the cellular repair response suggests that an inhibitor of MRN may sensitize tumors to agents that induce DNA breaks. Functional studies of the discrete molecular events involved in the detection of DSBs and the complex signal amplification events that ensue afterwards have been hampered by the embryonic lethality of the Mrella, Rad50 and Nbn gene disruptions. Although viable hypomorphic mutations in Mrell and $\mathrm{Nbs} l$ have been identified, the article by Dupré et al. ${ }^{1}$ reports the discovery of the first small-molecule inhibitor of MRN function that has uncovered a previously unappreciated role of the Mre11 nuclease in activation of the damage response to DSBs.

The central importance of the MRN complex in the processes of DNA damage signaling, genome stabilization, telomere length maintenance and meiosis was first elucidated genetically in yeast ${ }^{2}$, and further studies quickly identified homologs in other organisms, including humans. Mutations in the human Nbn and Mrella genes (NBN and MRE11A) cause the phenotypically similar genetic disorders Nijmegan breakage syndrome and ataxia telangiectasia-like disease, respectively. Both of these diseases give rise to genomic instability, hypersensitivity to radiation or to chemical agents that give rise to DSBs, and abnormalities in DNA damage cell-cycle checkpoints.

Structural and biochemical studies have begun to elucidate the architecture of the MRN complex and piece together the biochemical events that lead to activation of the cellular response to DSBs (Fig. 1a) ${ }^{3,4}$. The central player in the MRN complex is the Mre11 homodimer. Mre11 has the ability to bind free double-stranded DNA ends and also has an intrinsic $3^{\prime}-5^{\prime}$ exonuclease activity that serves to resect broken DNA ends. A key aspect of the molecular architecture is the interaction of the Mre11 dimer with two molecules of Rad50 and with the scaffolding protein Nbs1 (Fig. 1a). The extended coiled-coil domains of Rad50 allow it to extend out from DNA breaks and bridge with another Rad50 protein via a $\mathrm{Zn}^{2+}$-binding CXXC 'hook' motif. Thus, through its interaction with Mre11, Rad50 may serve as a molecular tether to bridge two broken DNA ends.

A key signaling role of the MRN complex is the recruitment and activation of the ATM (ataxiatelangiectasia mutated) kinase that then begins a wave of phosphorylation events that lead to signal amplification, cell cycle arrest and DNA repair (Fig. 1a). In this issue, Dupré et al. use a powerful forward genetic screen to discover a small-molecule inhibitor of the MRN-ATM pathway (mirin, Fig. 1b) from a 10,000-compound small-molecule library ${ }^{1}$. The screen 
involved the introduction of HaeIII-digested plasmid DNA (a mimic of DNA DSBs) into cellfree extracts obtained from Xenopus laevis eggs. The readout of the approach relied on phosphorylation of a peptide derived from the natural ATM substrate histone H2AX, which allowed detection of compounds that disrupt any step of the pathway leading up to $\mathrm{H} 2 \mathrm{AX}$ phosphorylation. Once the activity of mirin was detected in initial screens, its specificity for the ATM kinase pathway was demonstrated by immunodepleting ATM from extracts and showing that the phosphorylation of other downstream targets of ATM kinase is also inhibited by mirin.

So what is the molecular target of mirin? One obvious possibility is the ATM kinase itself. However, the authors clearly demonstrated that mirin inhibits only MRN-dependent ATM kinase activity, but has no effect on MRN-independent ATM activity, which strongly suggests that mirin does not interfere with the kinase itself. In determining the specific effect of mirin on the MRN complex, the authors systematically excluded inhibitory mechanisms involving disruption of (i) Mre11 or ATM binding to DNA, (ii) MRN-mediated DNA tethering and (iii) association of the MRN subunits. With possible targets running out, the authors then checked whether the exonuclease activity of Mre11 might be the mark. In support of this mechanism, in vitro experiments with Mre11 revealed that mirin inhibits Mre11 nuclease activity with a concentration dependence consistent with the inhibitory response observed in the H2AX phosphorylation assay. Thus, this extensive molecular detective work led to the conclusion that mirin inhibits ATM kinase activity indirectly by binding to Mre11 and disrupting its nuclease activity (Fig. 1b).

Given the known phenotypes of MRN hypomorphic mutations, it would be expected that a small molecule like mirin would inhibit cell cycle arrest and homology-dependent recombination (HDR) in human cells. Consistent with these expectations, mirin was found to abolish the ionizing radiation-induced G2/M cell cycle checkpoint in HEK 293 cells, and it also significantly decreased the occurrence of HDR-dependent gene conversion events. Thus, MRN is at the nexus of two cellular responses to DSBs, both of which are disrupted by mirin's inhibition of Mre11's nuclease activity.

The immediate impact of this work is the identification of the first small molecule that allows rapid inhibition of the Mre11 nuclease activity in cells, thereby bypassing the lethality associated with genetic disruptions of MRN function. Thus, the stage is set for the use of this new tool in the molecular dissection of other nuclease-requiring functions of the MRN complex in vitro and in living cells. In addition, specific inhibition of DNA damage repair and signaling pathways represents an exciting new avenue of drug discovery. For example, cells that have lost BRCA1 and BRCA2, components of the homologous recombination DNA repair pathway, are extremely sensitive to inhibitors of another damage-signaling enzyme-poly-ADP-ribose polymerase (PARP), which leads to selective cancer cell death. Thus, PARP inhibitors have already shown effectiveness in targeting BRCA-deficient breast cancers 5 . There is reason to anticipate that drugs targeting the MRN complex might also serve as valuable adjuvants to cancer therapy treatments by preventing tumor cells from detecting and efficiently repairing DNA breaks induced by ionizing radiation or chemical agents.

\section{References}

1. Dupré A, et al. Nat. Chem. Biol 2008;4:119-125. [PubMed: 18176557]

2. Stracker TH, Theunissen JW, Morales M, Petrini JH. DNA Repair (Amst) 2004;3:845-854. [PubMed: 15279769]

3. Hopfner KP, et al. Nature 2002;418:562-566. [PubMed: 12152085]

4. de Jager M, et al. Mol. Cell 2001;8:1129-1135. [PubMed: 11741547]

5. Ratnam K, Low JA. Clin. Cancer Res 2007;13:1383-1388. [PubMed: 17332279] 


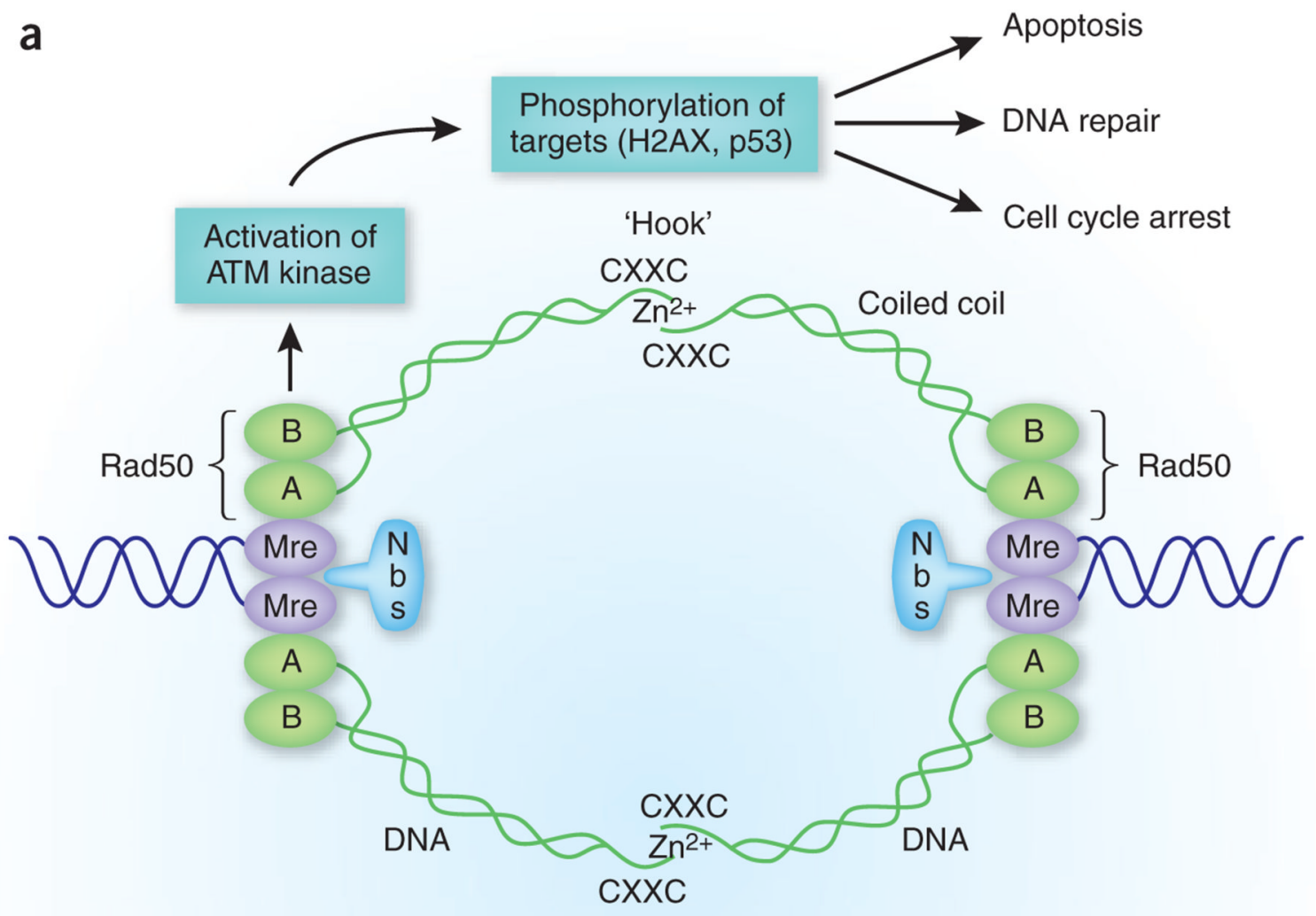

b

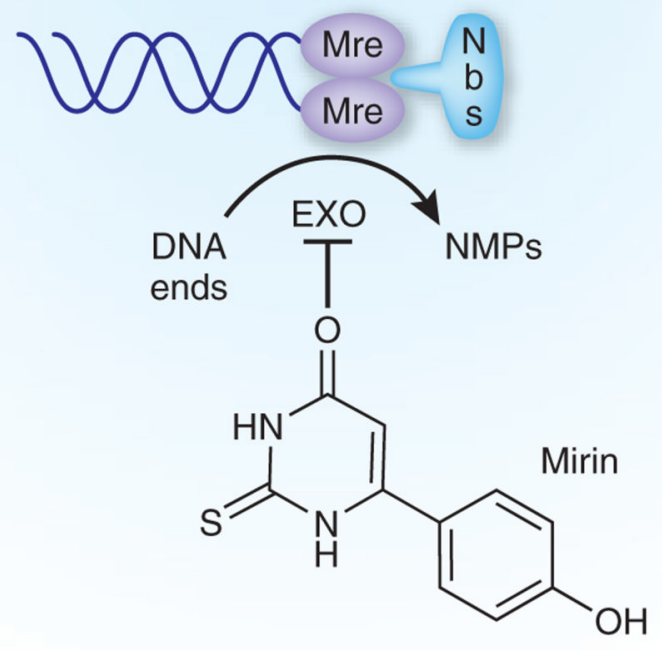

Figure 1. Architecture of the MRN complex bound to DNA DSBs and the proposed inhibition mechanism of mirin

(a) The MRN complex serves to bridge DNA breaks and signal the presence of DNA damage through the action of the ATM kinase pathway. Two of the downstream phosphorylation targets of ATM are indicated. The three components of the MRN complex are (1) Rad50, with its walker A and B ATP binding motifs, coiled-coil domains and $\mathrm{Zn}^{2+}$-binding CXXC hook motif, (2) the Mre11 homodimer, which has $3^{\prime}-5^{\prime}$ exonuclease activity on DNA ends, and (3) the Nbs1 scaffold protein. The location of the Nbs1 protein is speculative. (b) Mirin has been shown to inhibit the 3'-5' exonuclease activity of Mre11 (EXO) and block the activation of the ATM pathway. NMPs, nucleotide monophosphates. 